\title{
Kiefergelenkfortsatzbasis- und Kiefergelenkhalsfrakturen bei Kindern und Jugendlichen - Plädoyer für eine transoral endoskopisch assistierte operative Versorgung bei starker Dislokation und Luxation
}

\author{
Sebastian Schiel, Florian Probst, Peter Mayer, Gabriele Kaeppler, Carl Peter Cornelius
}

\section{Zusammenfassung}

Während bei Erwachsenen weitgehend klare Indikationen für die operative Versorgung von Kiefergelenkfortsatzfrakturen bestehen und diese Frakturen mehrheitlich operativ versorgt werden, wird bei Kindern und Jugendlichen immer noch ein nicht operatives (konservatives) Behandlungskonzept bevorzugt. Argument dafür ist das vermeintlich hohe spontane Regenerationspotenzial bis ins frühe Teenageralter. Stark dislozierte ( $>45^{\circ}$ Achsabweichung) und luxierte Kiefergelenkfortsatzbasis- und Kiefergelenkhalsfrakturen, die nicht operativ behandelt wurden, führen bei Kindern und Jugendlichen nicht selten zu Wachstumsstörungen mit Gesichtsasymmetrien, Bissstörungen und Dysplasien im Kiefergelenkbereich. Aktuelle Therapiekonzepte tendieren deshalb auch bei Kindern und Jugendlichen mit Luxationen oder erheblichen Dislokationen zu einer operativen Versorgung mit anatomisch korrekter Reposition und rigider Fixierung. Dieser Paradigmenwechsel von geschlossener Behandlung zur Osteosynthese über einen trans- fazialen oder insbesondere den hier dargestellten transoralen Zugangsweg hat sich erst auf der Grundlage von Fortschritten in der Behandlung von Kiefergelenkfortsatzfrakturen bei Erwachsenen entwickelt. Im Folgenden werden Überlegungen zu einem Klassifikationsvorschlag für pädiatrische Kiefergelenkbasis- und Kiefergelenkhalsfrakturen, die typische klinische Symptomatik einschließlich der bildgebenden Diagnostik und das derzeitige Behandlungskonzept mit dem operativtechnischen Vorgehen dargestellt.

\section{Condylar Base and Neck Fractures} in Children and Adolescents - A Plea for Transoral Endoscopically Assisted Management of Severe Dislocations and Luxations

While indications for the surgical treatment of condylar base and neck fractures in adults are rather well defined with most fractures being treated by open reduction and fixation, the preference in children and adolescents is still for non-operative therapy. The argument in favour of this is the presumably high remodelling potential up to the early adolescent age. However, non-operative treatment of severely displaced and dislocated condylar neck and base fractures in children and adolescents may result in growth disturbances with facial asymmetry, malocclusion and dysplastic shape of the condylar process. Thus, present day concepts suggest, even for these age groups, the use of open surgical treatment for displaced and dislocated condylar neck and base fractures with anatomic reduction and rigid fixation. The paradigm shift from closed treatment to open reduction and osteosynthesis using a transfacial or, as emphasised here, a transoral approach could be developed only on the basis of recent advances in the treatment of condylar process fractures. Some basic thoughts about a classification proposal for paediatric condylar base and neck fractures, the clinical symptomatology including diagnostic imaging and an actual treatment concept, emphasising a transoral endoscopically assisted approach and its surgical technique are presented in this article.

\section{Einleitung}

Im Kindes- und Jugendalter tritt der überwiegende Anteil der Unterkieferbrüche im Kiefergelenkbereich auf (Rowe 1969, Lindahl 1977, Thoren et al. 1997, Iatrou et al. 2010).

Gegenwärtig wird allgemein in den Altersgruppen bis 14 Jahre ein nicht operatives (konservatives) Behandlungskonzept mit der folgenden Argumentation

OP-JOURNAL 2013; 29: 156-163

(c) Georg Thieme Verlag KG Stuttgart · New York DOI http://dx.doi.org/10.1055/s-0033-1350818 bevorzugt: Kinder- und Jugendliche bis ins junge Teenageralter besitzen ein hohes Regenerationspotenzial mit Remodelling und funktioneller Adapation im Kiefergelenkbereich. Gleichzeitig würde eine operative Therapie die Gefahr der Schädigung des motorischen Gesichtsnervs mit sich bringen sowie möglicherweise eine Resorption des Kondylus und äußerlich sichtbare Narben im Gesicht hinterlassen (Rowe 1969, Kaban et al. 1977, Lindahl et Hollender 1977, Strobl et al. 1999, Lekven et al. 2011).

Bei stark dislozierten und luxierten Kiefergelenkfortsatzbasis- und Kiefergelenkhalsfrakturen kann eine vollständige
Wiederherstellung der Kiefergelenkfunktion jedoch mit einer nicht operativen Behandlung nicht erreicht werden.

Solche in Fehlstellung verheilten Frakturen können zu Hypo- und Dysplasien der Kiefergelenkregion mit Verminderung der vertikalen Höhe des aufsteigenden Unterkieferasts führen und damit einhergehend eine eingeschränkte Gelenkfunktion, Wachstumsstörungen und Gesichtsasymmetrien zur Folge haben (Kaban et al. 1977, Cornelius et al. 1991, Pirttiniemi et al. 2009).

Im Gegensatz dazu wird über gute funktionelle und morphologische Resultate 


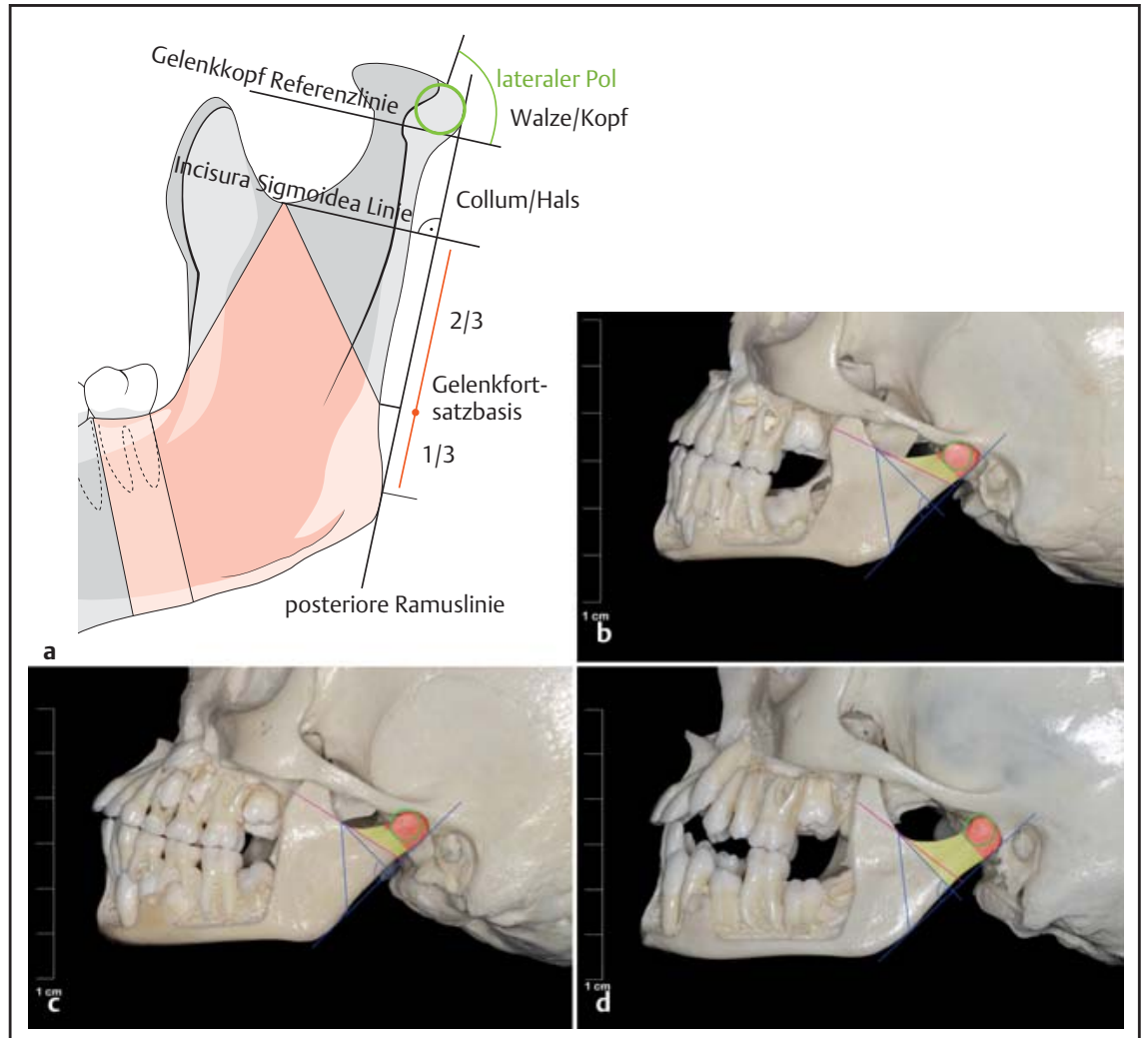

Abb. 1 a bis d AO-Klassifikation der Kiefergelenkfortsatzfrakturen für Erwachsene in Gegenüberstellung zu 3 Entwicklungsstadien des Unterkiefers vom Milchgebiss bis in die Spätphase des Stützzonenwechsels. Die Skelettpräparate sind zum Größenvergleich maßstabsgerecht fotografiert. a Lateralansicht des adulten R. ascendens im Schema - die auf der posterioren Ramuslinie im Lot stehende Incisura-sigmoidea-Linie trennt die Gelenkfortsatzbasis vom Gelenkhals bzw. Kollum; zur Abgrenzung der Gelenkwalze vom Kollum wird eine Tangente kaudal an einen Kreis (grün) gelegt, der den lateralen Pol der Walze umgibt; b Unterkiefer mit Milchgebiss im Alter von $\geq 2 \frac{1}{2}$ bis zu maximal 5 Jahren - Divergenz von anatomischen Komponenten ( $r o t=\mathrm{Ka}$ pitulum, gelb = Schaft, rote Linie = Tangente am tiefsten Punkt der Inzisur) und geometrischen Referenzlinien (blau); näheres siehe Text; c Unterkiefer nach Einstellung der 6-Jahr-Molaren und Durchtritt der Schneidezähne im Alter von ca. 7-8 Jahren - Immer noch die zuvor beschriebene Divergenz; d Unterkiefer gegen Ende des Stützzonenwechsels nach komplettem Abtausch der Milchzähne im Alter von etwa 10-11 Jahren - zunehmende Koinzidenz von anatomischen Komponenten mit den Referenzlinien beim Erwachsenen.

nach operativer Behandlung von stark dislozierten Kiefergelenkfortsatzbasisund Kiefergelenkhalsfrakturen bei Kindern, Jugendlichen (Schön et al. 2005, Deleyiannis et al. 2006, Iatrou et al. 2010, Schiel et al. 2013) und Erwachsenen (Ellis III et al. 2000, Schneider et al. 2008, Schmelzeisen et al. 2009) berichtet.

Zur Exposition der Kiefergelenkfortsatzbasis und des Kiefergelenkhalses wurden verschiedene operative Zugänge von präaurikulär, ohne oder mit temporaler Extension, retromandibulär/retrotrans-, anteparotideal, retroaurikulär/ transmeatal, submandibulär bis periangulär - beschrieben. Diese transfazialen, äußeren Zugänge zum Kiefergelenkfortsatz sind mit mehr oder weniger auffälligen äußerlich sichtbaren Narben im Gesicht, dem potenziellen Risiko einer lichten eine Renaissance in den letzten beiden Jahrzehnten. Inzwischen konnten zahlreiche Studien die Vorteile der transoralen Vorgehensweise für Erwachsene (Fritzemeier et Bechtold 1993, Jacobovicz et al. 1998, Schön et al. 2005, Schmelzeisen et al. 2009) sowie für Kinder und Jugendliche (Schön et al. 2005, Schiel et al. 2013) belegen.

\section{Zur Klassifikation von Kiefergelenk- fortsatzfrakturen vom Kindes- bis ins frühe Teenageralter}

Gelenkfortsatzfrakturen des Unterkiefers können auf unterschiedlichem HöhenLevel des Ramushinterrands ohne und mit Dislokation und/oder Luxation des gelenktragenden Fragments auftreten. Eine Klassifikation gibt es aktuell nur für Erwachsene (Schiel et al. 2012). Diese Erwachsenenklassifikation (Abb. 1 a) unterscheidet folgende Frakturtypen

- Gelenkfortsatzbasisfrakturen

- Gelenkhals- bzw. „Collum“-Frakturen

- Gelenkwalzenfrakturen (Synonyme: Gelenkkopf oder diakapituläre Frakturen)

Ein Klassifikationsvorschlag für Kinder und Jugendliche müsste an den Entwicklungsstadien des Unterkiefers unter dem Einfluss von Zahndurchbruchszeiten, Zahnwechsel (Milchdentition, Ersatzund Zuwachszahnung, vollständige bleibende Dentition) (Abb.1 b-d), Wachstumsfortschritten des Gesichtskeletts sowie letztlich am chronologischen Alter orientiert werden. Bis $\mathrm{zu}$ einem Alter zwischen 6 und 7 Jahren weist der kindliche Unterkiefer in der Seitenansicht allenfalls ein rudimentär ausgebildetes Collum auf, dessen Aussehen nicht der Sanduhr- bzw. eingezogenen Konusform beim Erwachsenen entspricht.

Im Wachstumsverlauf kommt es parallel zum appositionellen Wachstum in Oberkiefer und Gesichtschädel sowie zur Weichgewebeexpansion kontinuierlich zu relativen Lageänderungen (Relokation) der Unterkieferspange weg von den beiden Gelenkverbindungen an der Schädelbasis nach vorne unten. Die neue Lagebeziehung setzt intensive Remodellierungsprozesse in Gang, um den Unterkiefer in Form und Größe anzupassen. Im aufsteigenden Ast ( $R$. ascendens) ist das adaptive Remodelling dabei nach hinten oben gerichtet. Während der Ramus an der Vorderkante abgebaut wird und sich sowohl nach dorsal und lateral ausdehnt, nimmt die Seitenlänge der 


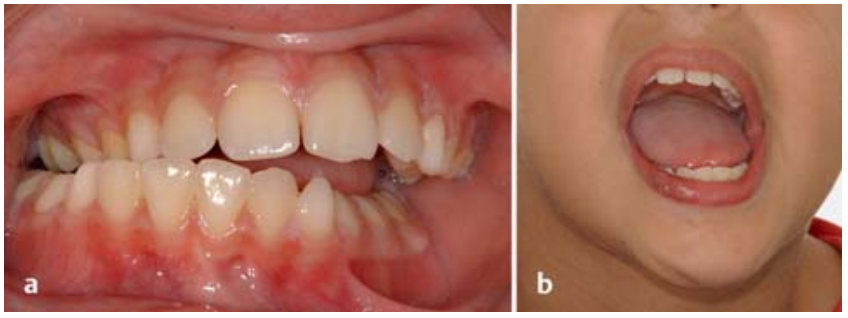

Abb. 2a und b Kiefergelenkfortsatzbasisfraktur rechts bei einem 7jährigen Jungen. a Anterolateral offener Biss auf der Gegenseite links; alleiniger Zahnkontakt im Seitenzahngebiet rechts; b Seitabweichung bei Öffnung des Unterkiefers, eingeschränkte Maximalöffnung. Die folgenden Abbildungen zeigen den gleichen Patienten.

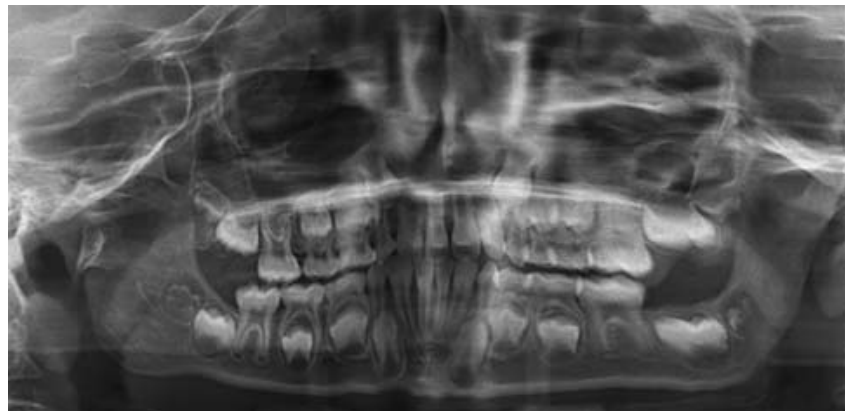

Abb. 3 Paromaschichtaufnahme (PSA) präoperativ. Kiefergelenkfortsatzbasisfraktur rechts (gleicher Patient wie in Abb. 2).
Unterkieferspange um den Betrag der Relokation zu, um auf dem neu angelegten Balkon (Tuberositas lingualis) Raum für die bleibenden Molaren zu schaffen.

Ab einem Alter von 2-21/2 Jahren entwickelt sich aus dem unteren Teil des plumpen, der Kondylusbasis fast direkt aufsitzenden Gelenkkopfs (Abb. 1 b) zunehmend eine Art Schaft.

Gleichzeitig mit dem Anstieg der Vertikalhöhe des Ramus beginnt sich die bis dahin flache Einbuchtung zwischen Gelenk- und Muskelfortsatz (Incisura sigmoidea oder mandibulae) auszurunden und zu vertiefen, wobei sich der R. ascendens auch in die Sagittalebene ausdehnt.

Mit 7-8 Jahren wird erstmals ein etwas längerer Gelenkhals im Sinne einer Übergangszone zwischen Basis und Gelenkkopf identifizierbar, die Vertikalhöhe des Ramus hat wie die Breite der Unterkieferspange und Größe des Molarenfelds durch die Wanderung der 6-JahrMolaren in die okklusale Kontaktbeziehung weiter zugenommen (Abb. 1 c).

Im Alter von 10-11 Jahren mit dem Zahnwechsel in den Stützzonen (= Bukkalzone zwischen seitlichem Schneidezahn und 6-Jahr-Molaren) (Abb.1 d) erreicht der Mandibularbogen bereits näherungsweise die spätere Transversaldimension. Während der Wachstumsfortschritt der Alveolarfortsätze in Oberund Unterkiefer noch für die weitere Entwicklung des Angulus/Ramus entlang der Vertikalachse und in der Sagittalebene sorgen muss, sind die definitiven Proportionen von Muskel- und Gelenkfortsatz zueinander schon soweit hergestellt, dass sich die RemodellingAktivität in den Processus verlangsamen kann.

Ähnlich wie beim Erwachsenen ist nunmehr ein distinktes Collum vorhanden, das sich in den Folgejahren mit Abschluss der bleibenden Dentition und weiterer Zunahme der Gesichtshöhe konisch verjüngt.

Die Verwendung der gleichen Landmarken und Bezugslinien, mit denen die Topografie bzw. die Frakturhöhe am Gelenkfortsatz von Erwachsenen bestimmt wird (Abb. 1 a), zeigt bei Übertragung auf sukzessive Entwicklungsstadien des Gesichtskeletts (Abb.1 b bis d) einmal mehr, dass es sich bei der Mandibula von Kindern und Jugendlichen nicht einfach um Miniaturausgaben eines Erwachsenenunterkiefers handelt. Zwar ergibt sich aus der Konstruktion der Incisura-sigmoidea-Linie im Lot zur posterioren Ramuslinie sowohl im Alter von 21/2 Jahren (Abb. $\mathbf{1}$ b) als auch im Alter von etwa 8,5-9 Jahren (Abb. 1 c) ein breitbasiges rechtwinkliges Dreieck, das jeweils Vexierbilder eines gut ausgeprägten $\mathrm{Ge}$ lenkfortsatzes vermittelt. Das entspricht unseren Sehgewohnheiten und „man sieht, was man sehen möchte“. Aber de facto ist die Incisura sigmoidea noch sehr flach und dementsprechend sind der Muskel- und der Gelenkfortsatz sehr kurz. In einem rasanten Wachstumsschub ändern sich dann die Dimension und Formgebung des muskel- und des gelenktragenden Fortsatzes.

Diese beiden Untereinheiten (muskelund gelenktragender Fortsatz) des Ramus dürften ungefähr zwischen dem 9. und 10. Lebensjahr (übrigens wie die Augenhöhle), spätestens aber im frühen Teenageralter ihre definitive Größe angenommen haben und Skelettelemente darstellen, die im Weiteren nur noch räumlich verlagert werden.

In diesem Altersabschnitt fallen die anatomischen Gegebenheiten und die geometrischen Referenzen immer näher zusammen. Darin kommt der Abschluss des intensivsten Wachstumsgeschehens bereits in der späten Kindheitsphase zum Ausdruck und die Frakturklassierung im Processus condylaris kann analog zum Erwachsenen vorgenommen werden. Vermessungen an einer umfangreichen Schädelsammlung aus dem Anatomischen Institut der Universität Würzburg (35 Kinder bis zu 10 Jahren, 51 Erwachsene) ergab in der Vertikalhöhe der Gelenkfortsätze (Differenz Ramushöhe insgesamt minus Höhe bis zum tiefsten Punkt der Incisura sigmoidea $=15 \mathrm{~mm}$ ) ab der Altersgruppe zwischen 7 und 10 und Erwachsenen keine Unterschiede (Lang und Öder 1984).

In einer „Pädiatrischen“ Klassifikation von Kiefergelenkfortsatzfrakturen sollten, abhängig vom Dentitionsalter und in Hinsicht auf die Wachstumsintensität, 3-4 Entwicklungsstadien Berücksichtigung finden.

Von einem Collum sollte frühestens ab dem 7. bis 8. Lebensjahr die Rede sein.

Im Alter davor sollte ein anderer Begriff zur Bezeichnung der Substruktur unterhalb des Gelenkkopfs bis auf Höhe der Incisura sigmoidea gesucht werden. Beispielsweise bieten sich an Kondylusschaft, Stamm, Wurzel - bzw. Scapus, Truncus, Radixshaft, Stock, Trunk, RootRCPT (Ramus Condylar Process Transition) -, treffen den Sachverhalt aber noch nicht genau. Die Begriffsbildung aber ist nur ein Punkt einer Klassifizierung (Cornelius et al. 2013). Selbstverständlich müssen Frakturlinienlokalisation und -verläufe mit der Bildgebung aus Patientenfällen korreliert und ggf. den realen Gegebenheiten angepasst werden. Unter anderem ist bei den in diesem Beitrag nicht erörterten, pädiatrischen Kapitulumfrakturen in frühen Entwicklungsstadien nicht mit Gewissheit klar, ob sie wie beim Erwachsenen an der Sagittalebene ausgerichtet sind oder ein anderes Muster aufweisen. Au- 

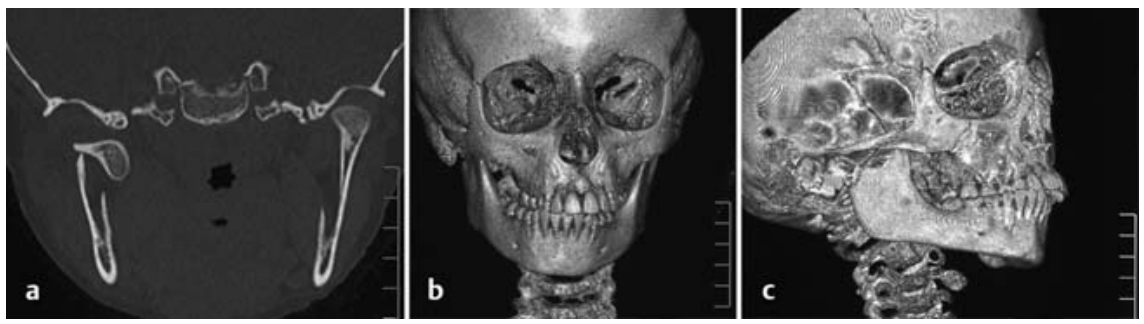

Abb. 4a bis c CT-Diagnostik präoperativ. a Koronale Schicht im Bereich des Ramushinterrands bzw. der Kiefergelenkfortsätze - Schrägfraktur des Gelenkfortsatzes rechts auf Höhe der Basis, um $90^{\circ}$ nach medial disloziertes gelenktragendes Fragment mit kompletter Luxation des Gelenkkopfs aus der Fossa; b 3-D-CT von frontal - Seitabweichung des Unterkiefers zur Frakturseite; c 3-D-CT von anterolateral rechts: Dislokation des Gelenkfortsatzes nach medial - Ansicht auf die Bruchfläche des gelenktragenden Fragments, leere Gelenkpfanne aufgrund der Luxation (gleicher Patient wie in Abb. 2 und 3).

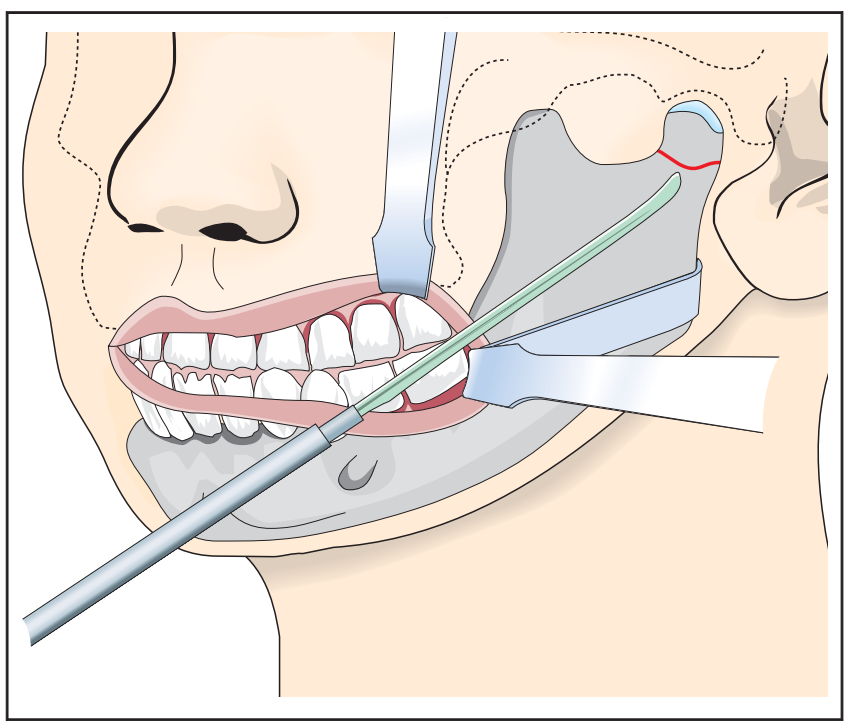

Abb. 5 Transoraler Zugang zur Kiefergelenkregion - Verhältnisse im Alter von ca. 9 Jahren. Der Gelenkfortsatz, v.a. die Übergangsregion Ramus/Processus condylaris, ist relativ leicht zu erreichen.

ßerdem wünschenswert wäre eine an moderne technische Möglichkeiten adaptierte 3-D-Betrachtungsweise, womit sich die grundsätzliche kritische Frage stellt, ob der aufsteigende Unterkieferast - ausgenommen in der Lateralansicht - überhaupt eine Form aufweist, die den Namen Collum verdient.

\section{Klinische Symptome}

Erster Hinweis für das Vorliegen von Kiefergelenkfortsatzfrakturen nach Stürzen im Kindesalter sind Platz- oder RissQuetsch-Wunden im Kinnbereich.

Typische Leitsymptome sind dann Okklusionsstörungen:

- Bei einseitiger Fraktur besteht kontralateral und im Frontzangebiet ein offener Biss. Beim Zusammenbeißen bzw. in Interkuspidationsstellung sind Zahnkontakte alleinig im Seitenzahngebiet der Frakturseite festzustellen. Zudem weicht der Unterkiefer insgesamt zur Frakturseite ab (Abb. 2).
- Bei beidseitigen Frakturen besteht ein frontal offener Biss. Die Zähne haben nur im Molarenbereich Kontakt. Der Unterkiefer rutscht in eine Retrallage mit Verlust der hinteren Gesichtshöhe.

Die Okklusionsstörungen erklären sich zung des aufsteigenden Unterkieferasts durch den Zug der umgebenden Kaumuskelschlinge (M. masseter und $M$. pterygoideus medialis).

Zusätzlich können die Kieferöffnung und die Vorschub- und Seitwärtsbewegungen eingeschränkt sein, weil der unterhalb des Kieferköpfchens ansetzende $M$. pterygoideus lateralis durch die knöne Zugwirkung auf die Unterkieferspange verliert. Präaurikulär und im oberen Bereich der Parotis imponieren oft deutlich sichtbare Schwellungen. Bei Palpation sind sie äußerst schmerzhaft, da Einblutungen im Gelenkkapselbereich aus der Dislokation mit Längsverkürcherne Unterbrechung kaudal davon sei- vorliegen und/oder die Fragmenten ins Weichgewebe einspießen. Schon vorsichtiger Druck in dorsokranialer Richtung löst dann heftige Schmerzen aus.

Bei Luxationsfrakturen (= Kieferköpfchen außerhalb der Fossa articularis) tastet sich die Gelenkgrube leer. Bei medialer oder anterokaudaler Dislokation des gelenktragenden Fragments ist der laterale Walzenpol im Ablauf einer Kieferöffnungsbewegung nicht mehr durchtastbar.

Gelegentlich blutet es aus dem Ohr, wenn die Vorderwand des äußeren Gehörgangs einschließlich der Haut nach einem Aufprall auf das Kinn perforiert ist.

\section{Bildgebende Diagnostik}

Die Panoramaschichtaufnahme (PSA) wird auch bei Kindern zum Screening bzw. zur Basisdokumentation von Frakturen im Ober und Unterkiefer eingesetzt. (Abb. 3). Die Ergänzung der PSA mit mindestens einer 2. (senkrecht orientierten) Darstellungsebene, z.B. im okzipitofrontalen Strahlengang (Clementschitsch-Projektion) gilt immer noch als Minimalstandard. Die Computertomografie (Abb. 4). oder die digitale Volumentomografie (DVT) (Kaeppler et al. 2013) ermöglicht jedoch eine weit detailliertere, multiplanare (koronal, axial, sagittal) Beurteilung der Frakturzonen sowie die 3-dimensionale Rekonstruktion der Kiefergelenkfortsätze, der Fossae articulares, mitsamt den Gehörgangswänden wie auch des übrigen $\mathrm{Ge}$ sichtsskeletts.

In puncto Aussagekraft und Genauigkeit gibt es daher zzt. keine sinnvolle Alternative zu den modernen digitalen Schnittbildverfahren (CT oder DVT).

\section{Behandlungskonzept}

Die Wiederherstellung des Gelenkfortsatzes in originärer anatomischer Konfiguration und die Reetablierung prätraumatischer okklusaler Verhältnisse bieten unbestritten ideale Voraussetzungen für die Rückkehr ungestörter Funktionsabläufe und die weitere Entwicklung des Unterkiefers bzw. Gesichtsskeletts.

Nicht operative (geschlossene) Therapieansätze ohne Reposition stark dislozierter oder luxierter gelenktragender Fragmente müssen dagegen auch bei Kindern mit Remodellierungs-Potenzial und 
gerade bei Jugendlichen im frühen Teenageralter zwangsläufig kompromissbehaftet sein. So wäre zur knöchernen Ausheilung der Kiefergelenkfortsatzbasis- und -gelenkhalsfraktur nach geschlossener Reposition (sofern das überhaupt machbar ist) eine Ruhigstellung des Unterkiefers von mindestens 3-4 Wochen Dauer erforderlich. Allerdings ist eine Fragmentreposition bei extremer Achsenknickung (Dislocatio ad axim) und/oder Verkürzung (Dislocatio ad latus cum contractione bzw. lateral oder medial override) ggf. sogar mit Kontaktverlust der Fragmentenden konservativ nicht zu bewerkstelligen, und eine Retention bis zur Konsolidierung in Fehlstellung wäre kontraproduktiv. Damit es zur Ausbildung von Nearthrosen (Rasse 2000) oder auch Pseudarthrosen unter muskulärer Führung kommen kann, wird eine Frühmobilisation nach vergleichsweise kurzen Immobilisationsphasen von maximal 10-14 Tagen nötig. Bei hochlokalisierten Walzenabriss- oder Walzenfrakturen ist eine Wiederabstützung des Ramus in der vertikalen Ausgangsdimension evtl. leichter zu erreichen.

Konservative Therapieansätze bei Kiefergelenkfortsatzbasis- und -gelenkhalsfrakturen mit temporärer Immobilisation und anschließenden Funktionsübungen sind deshalb nur bei nicht oder geringfügig dislozierten stabilen Fragmenten Erfolg versprechend und allenfalls in besonderen Konstellationen (bspw. OP-Kontraindikation aufgrund schwerwiegender Allgemeinerkrankungen, operative Therapie wird vom Patienten abgelehnt) zu rechtfertigen.

Über Wachstumsstörungen mit Ausbildung von mandibulären Laterognathien, Kiefergelenksdegeneration und Gesichtsasymmetrie bei Kindern und Jugendlichen als Folge von nicht operativ behandelten Kiefergelenkfortsatzfrakturen wurde mehrfach berichtet (z.B. Kaban et al. 1977, Pirttiniemi et al. 2009). Ein Review über konservative Behandlungskonzepte bei Kiefergelenkfrakturen im Kindes- und Jugendalter kommt zu dem Ergebnis, dass sich das Risiko für ein ungünstiges radiologisches und funktionelles Outcome bei starkem Dislokationsgrad, tiefem Frakturlinienverlauf und zunehmendem Lebensalter erhöht (Dodson 2005).

Aufgrund technischer Fortschritte ist die operative Versorgung von Kiefergelenkfortsatzbasis-, -hals und -walzenfrakturen bei Erwachsenen auf dem Wege, sich

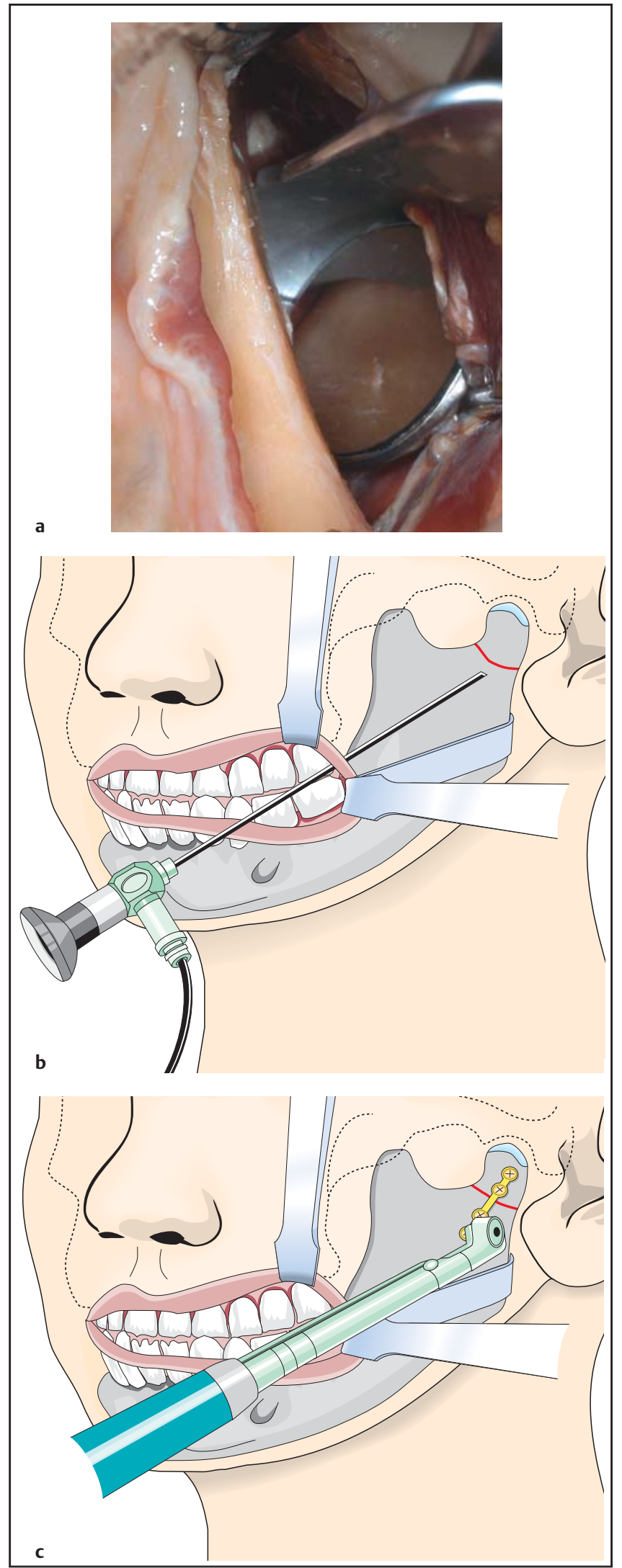

Abb. 6 a bis c

Reposition und Osteosynthese. a Kontrolle des Ramushinterrands links mit einem Zahnarztspiegel - Simulation am anatomischen Präparat; $\mathbf{b}$ endoskopische Kontrolle der Fragmentstellung am Ramushinterrand; c Fixation des gelenktragenden Fragments mit einer Mini-Osteosyntheseplatte - bei Verwendung von 2 Miniplatten wird, anders als hier gezeigt, zuerst eine kürzere Platte anterior unterhalb der Incisura sigmoidea angebracht, dann kann das Fragment am Hinterrand vor Anlegen der 2. Platte nachjustiert werden. als allgemeiner Standard durchzusetzen. Wenn Achsabweichungen zwischen $10^{\circ}$ und $45^{\circ}$ und Verkürzungen der Ramushöhe $\geq 2 \mathrm{~mm}$ vorlagen, hat sich die of- fene Reposition und interne Fixierung (ORIF) gegenüber einer geschlossenen Immobilisationsbehandlung in einer prospektiven Multicenterstudie als 

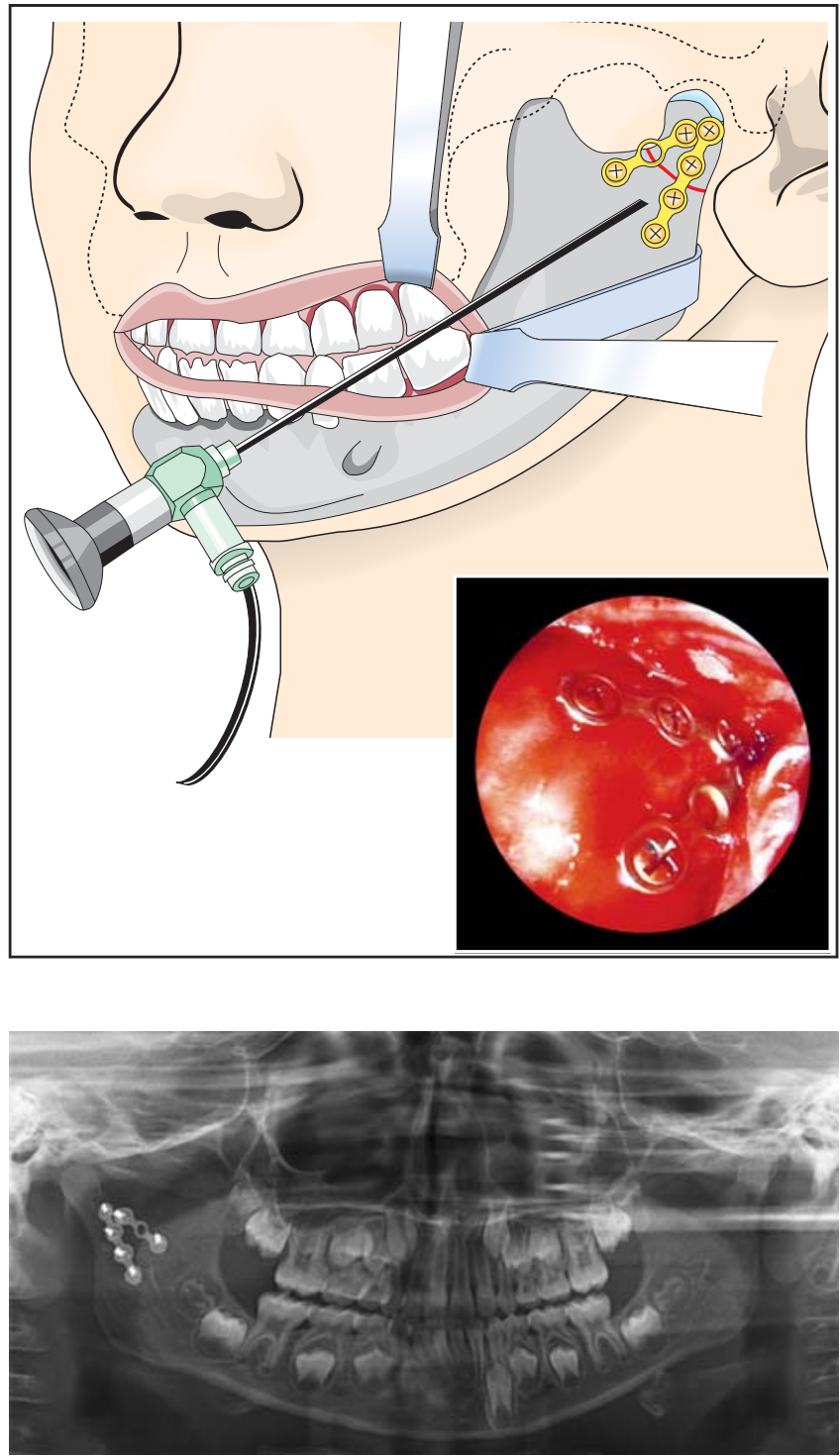

Abb. $7 \mathbf{a}$ und $\mathbf{b}$ a Schema der Fragmentfixation am Übergang Gelenkfortsatzbasis/-hals mit 2 Titan-Miniplatten; $\mathbf{b}$ Kontrolle nach Reposition und Plattenosteosynthese des Kiefergelenkfortsatzes rechts mit $30^{\circ}$ Endoskopoptik. Endoskopansicht - fertiggestellte Fixierung mit 2 Miniplatten am Vorder- und Hinterrand des Kiefergelenkfortsatzes (Patient aus Abb. 2 bis 4 und Abb. 8 bis 10).

Abb. 8 Postoperative Panoramaschichtaufnahme (PSA) des oben gezeigten 7 Jahre alten Jungen nach transoraler ORIF der Kiefergelenkfortsatzbasisfraktur rechts. überlegen erwiesen (Schneider et al. 2008).

Führt man sich neben diesen Ergebnissen die neuesten Berichte über ausgesprochen gute funktionelle und morphologische Resultate nach operativer Versorgung von Kiefergelenkfortsatzfrakturen im Kindes- und Jugendalter (Schön et al. 2005, Deleyiannis et al. 2006, Iatrou et al. 2010, Schiel et al. 2013) vor Augen (vgl. Abb. 7 bis 10), dann kann das nur ein Plädoyer für ein operatives Behandlungskonzept auch vor Abschluss des Wachstumsalters bedeuten - umso mehr, als Komplikationen vermieden werden können.

Die am meisten gefürchtete Komplikation bei Verwendung äußerer Zugänge zur operativen Versorgung der Gelenkfortsätze sind periphere Fazialisparesen. Neben der Vermeidung im Gesichtshaut- bereich sichtbarer Narben lässt sich dieses Risiko bei transoralen Vorgehensweisen deutlich minimieren (Fritzemeier und Bechtold 1993, Chen et al. 1998, Jacobovicz et al. 1998, Schön et al. 2005, Veras et al. 2007, Schmelzeisen et al. 2009).

\section{Transoral endoskopisch assistierter Zugang bei Kindern und Jugendlichen}

Wie beim Erwachsenen verläuft die typische Schleimhautinzision beim transoralen Zugang zum Kiefergelenkfortsatz entlang der Linea obliqua, d.h. über den tastbaren Vorderrand des aufsteigenden Unterkieferasts bzw. der Seitenkante des inneren Kieferwinkels. Nach vorne wird die Schnittführung an der mukogingivalen Grenze bis in die Milchmolaren oder Prämolarenregion fortgesetzt. Nach Eingehen auf die Knochenoberfläche wird die Lateralfläche des aufsteigenden Un- terkieferasts bis in den Bereich der Incisura semilunaris streng subperiostal dargestellt (Abb.5). Altersabhängig sind die anatomischen Verhältnisse deutlich graziler und die Distanzen über den knöchernen Oberflächen deutlich kürzer als im Erwachsenenalter. In kranialer Richtung wird die Gelenkfortsatzregion schnell erreicht. Nach dorsal wird der Hinterrand des R. ascendens freigelegt und der Frakturbereich unter Visualisierung mit einem Endoskop (30 $0^{\circ}$ HopkinsVorausblick-Optik + Video-Kamera-Monitor-System) soweit dargestellt, dass die Reposition und anschließende Osteosynthese des gelenktragenden Fragments möglich werden. Spezielle Retraktoren und - sofern es die Größenverhältnisse zulassen - der Einsatz beleuchteter Wundhaken mit Lichtleiterkabeln helfen dabei, die Übersicht zu verbessern. Vom Operateur sollte zudem eine Stirnlampe getragen werden. Mit einer Reihe von Spezialinstrumenten (Elevatoren, 90 Winkelbohrer und $90^{\circ}$-Winkelschraubendreher, Fasszangen) können in der Horizontalebene verlaufende Frakturen über den „transoral endokopisch assistierten Zugang“ (Fritzemeier et Bechtold 1993, Jacobovicz et al. 1998, Schön et al. 2005, Veras et al. 2007, Schmelzeisen et al. 2009) bis in einen Bereich von etwa $0,5-0,8 \mathrm{~cm}$ unterhalb des Kondylus versorgt werden.

Der Schwierigkeitsgrad steigt, je weiter kranial eine Fraktur lokalisiert ist, da der Exposition durch die zunehmend enger werdende und unnachgiebigere Weichgewebekavität Grenzen gesetzt sind.

Vor allem die Größendimensionierung der Bohrerköpfe wirkt sich limitierend aus.

\section{Reposition und Osteosynthese}

Die Reposition des Kiefergelenkfortsatzes wird mit speziellen (z. B. bajonettförmig, kellenartig, spiralig gebogen) Elevatorien unter gleichzeitiger Traktion am aufsteigenden Unterkieferast nach kaudal durchgeführt. Dazu wird eine Osteosyntheseschraube am Kieferwinkel inseriert, über die eine mit einer Ahle perkutan eingebrachte Drahtcerclage gelegt und zu einem Kabel verdrillt wird. Durch Zug am äußeren Kabelende kann dann die gewünschte Distraktion am Frakturspalt ausgeübt werden. Stattdessen kann auch versucht werden, eine Entlastung durch Druck auf die Molaren mit dem Daumen oder Zeigefinger zu erreichen. Bei starker Dislokation oder Lu- 


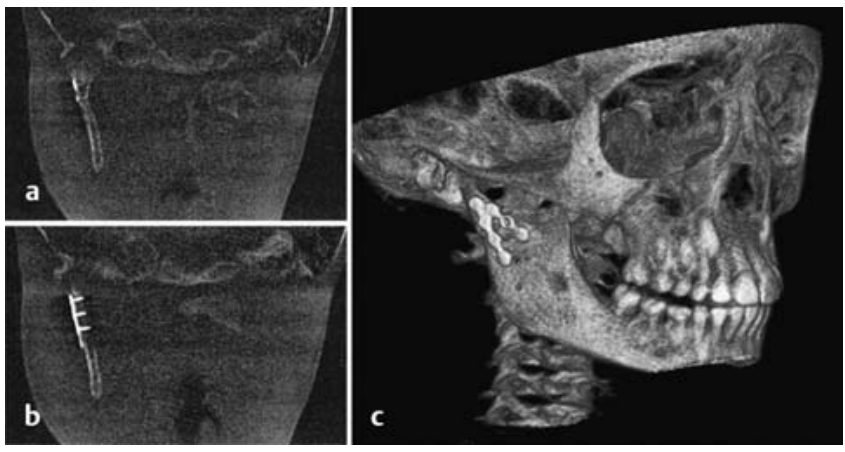

Abb. 9a bis c Postoperatives CT des zuvor gezeigten 7 Jahre alten Jungen $\mathbf{a}$ und $\mathbf{b}$. Koronare Schichten mit refixiertem gelenktragenden Fragment und Osteosynthesematerial in korrekter Achsenstellung; c Halbseitenprofil des Gesichtsschädels in 3-D-Reformatierung - wiederhergestellte normale Anatomie von Ramus und Gelenkfortsatz. Ein nennenswertes Collum ist in diesem Alter nicht immer vorhanden.

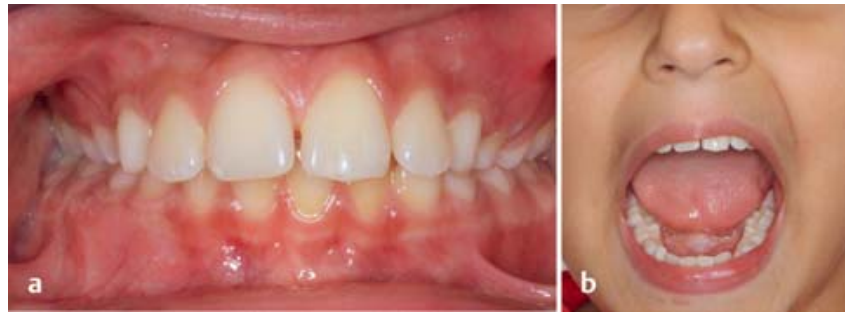

Abb. 10 a und b Klinische Befunde postoperativ. a regelrechte Okklusion; $\mathbf{b}$ uneingeschränkte Kieferöffnung. xationen haben einige gelenktragende Fragmente vermutlich keine Weichgewebeverbindung mehr und verhalten sich wie ein freies Transplantat. Die gleiche Situation besteht aber auch bei geschlossener Behandlung.

Sobald die Fragmente in achsengerechter Stellung reponiert sind, lässt sich die korrekte Position am besten am Hinterrand des aufsteigenden Unterkieferasts kontrollieren, entweder nach Einführen eines kleinen oder mittelgroßen zahnärztlichen Spiegels oder unter Benutzung der $30^{\circ}$-Endoskop-Optik (Abb. 6).

Eine vielfach bewährte Technik zur Fixation von Kiefergelenkfortsatzbasis oder -halsfrakturen ist die Osteosynthese mit Titan-Miniplatten. Wenn das Platzangebot dazu ausreicht, werden 2 Miniplatten benutzt (Abb. 6 und 7).

Diese werden in einer V-Stellung am Vorderrand unterhalb der Incisura sigmoidea und parallel zum Hinterrand angebracht (Abb. 7 bis 9). Dieses Osteosynthesemuster eignet sich auch bei Frakturen von Kindern und Jugendlichen. Zwecks besserer Übersicht wird zuerst eine kurze Adaptationsplatte am Vorderrand fixiert, die ein Abrutschen der Fragmente verhindert.

Nach nochmaliger Feinadjustierung und Überprüfung der Fragmentstellung (Abb. 7b) wird eine 2. längere (evtl. auch im Querschnitt stärkere) Adaptationsplatte vor dem Hinterrand platziert. In der 2-Platten-Anordnung werden pro Fragmentseite 3 Schrauben inseriert. Wenn ein schlanker Gelenkhals vorliegt, was im Kindes- und frühen Jugendalter eigentlich nicht zu erwarten ist, der eine Positionierung von 2 Platten nicht gestattet, sollte eine einzelne rigidere Platte Verwendung finden, wobei auch die sog. Grid- oder auch 3-D-Platten (Rhomboid, Delta, Lamda, Strut plate, etc.) infrage kommen. Jedoch ist bei der Auswahl Vorsicht geboten: nicht alle dieser zuletzt genannten Platten sind gleich gut zur transoralen Applikation und für die Größenverhältnisse im Kindesalter geeignet.

\section{Resümee}

Während bei Erwachsenen klare Indikationen für die operative Versorgung von Kiefergelenkfortsatzfrakturen bestehen (Valati et al. 2008), gehen die Präferenzen bei Kindern und Jugendlichen nach wie vor in Richtung einer konservativen Behandlung (Lindahl et Hollender 1977, Strobl et al. 1998, Lekven et al. 2011).

Hauptargument ist dabei die Behauptung, dass aufgrund des hohen Regenerations- und Remodelling-Potenzials im Wachstumsalter mit gezielter Physiotherapie eigentlich immer gute funktionelle Ergebnisse erreicht werden können.

Mehrere Studien zeigen jedoch in 5-10\% skelettale Defizite mit Gesichtsasymmetrien sowie ein dysplastisches Wachstum der aufsteigenden Unterkieferäste nach nicht operativer Behandlung von dislozierten Kiefergelenkfortsatzbasisund -gelenkhalsfrakturen in Kindheit und Jugend (Lund 1974, Lindahl 1977, Pirttiniemi et al. 2009) sowie eine hohe Prävalenz von Kiefergelenksfunktionsstörungen (Thoren et al. 2001, Strobl et al. 1999, Lekven et al. 2011).
Wenn aufgrund starker Dislokationen oder Luxationen bei Kiefergelenkfortsatzbasis- und -gelenkhalsfrakturen abzusehen ist, dass die Adaptationsleistung und Funktionswiederherstellung bei Anwendung eines nicht operativen Therapieprinzips nicht ausreichend sein wird, sind operative Behandlungsstrategien dringend indiziert, um skeletale Fehlstellungen und mögliche Folgeerscheinungen auszuschließen.

Mit zunehmender Skelettreifung (Jugend/frühes Teenageralter) nehmen die Kompensationsmöglichkeiten ab (vgl. Abb. 1), weshalb die Hemmschwelle $\mathrm{zu}$ einer operativen Vorgehensweise dann sinken sollte.

Nach unserer Einschätzung ist es nicht berechtigt, der offenen Reposition und Fixation von Kiefergelenkfortsatzbasisund -gelenkhalsfrakturen einen negativen Ruf anzulasten und sie von vornherein als überaggressiv abzulehnen.

Insbesondere in der Variante über einen transoralen Zugang und unter endoskopischer Assistenz kommen die Vorteile einer Wiederherstellung der originären Anatomie und damit einer ungestörten Gelenkfunktion ohne äußerlich sichtbare Narben sowie bei minimalem Risiko für Fazialisläsionen zum Tragen.

Mit Blick auf potenzielle Funktionsstörungen und die mögliche Wachtumsproblematik nach in Fehlstellung verheilten Kiefergelenkfortsatzbasis- oder Kiefergelenkhalsfrakturen bei Kindern und Jugendlichen, ist das Plädoyer, die ORIF bei starken Dislokationen und v.a. bei Luxationen, wenn immer möglich auf transoral endoskopisch assistiertem Weg, als festen Bestandteil im eigenen therapeutischen Spektrum zu etablieren, überfällig.

Selbstverständlich müssen sich die Ergebnisse operativ versus konservativ auf Basis einer verbindlichen Klassifikation zukünftig messen lassen. 


\section{Literatur}

${ }^{1}$ Chen CT, Lai JP, Tung TC et al. Endoscopically assisted mandibular subcondylar fracture repair. Plast Reconstr Surg 1998; 103: 160-165.

2 Cornelius et al. AO CMF Frakturen Klassifikation. OP Journal 2013; 29: 109-128

${ }^{3}$ Cornelius CP, Ehrenfeld M, Laubengeiger $M$ et al. Ergebnisse eines konservativ-funktionellen Therapiekonzepts bei kindlichen Kondylusfrakturen. Dtsch Zahnärztl Z 1991; 46: 46-49

${ }^{4}$ Deleyiannis FW, Vecchione L, Martin B et al. Open reduction and internal fixation of dislocated condylar fractures in children: longterm clinical and radiologic outcomes. Ann Plast Surg 2006: 57: 495-501

${ }^{5}$ Dodson TB. Condyle and ramus-condyle unit fractres in growing patients: management and outcomes. Oral Maxillofac Surg Clin North Am 2005; 17: 447-453

${ }^{6}$ Eckelt U, Schneider M, Erasmus F et al. Open versus closed treatment of fractures of the mandibular condylar process-a prospective randomized multi-centre study. J Craniomaxillofac Surg 2006; 34: 306-14

${ }^{7}$ Ellis III E, Throckmorton GS. Treatment of mandibular condylar process fractures: biological considerations. J Oral Maxillofac Surg 2005; 63: 115-134

8 Fritzemeier CU, Bechthold H. Die Osteosynthese von Unterkiefergelenkfortsatzfrakturen mit alleinigem Zugang von intraoral. Dtsch Z Mund Kiefer Gesichtschir 1993; 17: 66-68

${ }^{9}$ Hammer B, Schier P, Prein J. Osteosynthesis of condylar neck fractures: a review of 30 patients. Br J Oral Maxillofac Surg 1997; 35: 288-291

10 Iatrou I, Theologie-Lygidakis N, Tzerbos F. Surgical protocols and outcome for the treatment of maxillofacial fractures in children: 9 years' experience. J Craniomaxillofac Surg 2010; 38: 511-516

11 Jacobovicz J, Lee C, Trabulsky PP. Endoscopic repair of mandibular subcondylar fracture Plast Reconstr Surg 1998; 101: 160-165

12 Kaeppler G, Cornelius CP, Ehrenfeld M et al. Diagnostic efficacy of cone-beam computed tomography for mandibular fractures. Oral Surg Oral Med Oral Pathol Oral Radiol 2013; 116: $98-104$

${ }^{13}$ Lang J, Öder M. Über die Biomorphose der Mandibula. Gegenbaurs Morph Jahrb 1984; 130: 185-234

14 Lekven N, Neppelberg E, Tornes K. Long-term follow-up of mandibular condylar fractures in children. J Oral Maxillofac Surg 2011; 69: 2853-2859

15 Lindahl $L$. Condylar fractures of the mandible. I. Classification and relation to age, occlusion, and concomitant injuries of teeth and teethsupporting structures, and fractures of the mandibular body. Int J Oral Surg 1977; 6 : 12-21
${ }^{16}$ Lindahl L, Hollender L. Condylar fractures of the mandible. II. A radiographic study of remodeling processes in the temporomandibular joint. Int J Oral Surg 1977; 6: 12-21

17 Lindahl L. Condylar fractures of the mandible. III. Positional changes of the chin. Int J Oral Surg 1977; 6: 166-172

18 Loukota RA, Eckelt U, De Bont L et al. Subclassification of fractures of the condylar process of the mandible. Br J Oral Maxillofac Surg 2005; 43: 72-73

${ }^{19}$ Lund $K$. Mandibular growth and remodeling processes after condylar fracture. A longitudinal roentgencephalometric study. Acta Odontol Scand Suppl 1974; 32: 3-117

${ }^{20}$ Rasse M. Neuere Entwicklungen der Therapie der Gelenkfortsatzbrüche der Mandibula. Mund Kiefer Gesichtschir 2000; 4: S69-S87

${ }^{21}$ Rowe NL. Fractures of the jaws of children. J Oral Surg 1969; 27: 497-507

22 Schiel S, Smolka W, Leiggener $C$ et al. „Open book“-Frakturen des Mandibularbogens: Bilaterale Gelenkfortsatzfrakturen in Kombination mit Paramedian-/Medianfrakturen des Unterkiefers. Operative Behandlungsstrategien. OP Journal 2012; 28: 194-210

23 Schiel S, Mayer P, Probst FA et al. Transoral open reduction and fixation of condylar base and neck fractures in children and young teenagers - a beneficial treatment option? J Oral Maxillofac Surg 2013; 71: 1220-1230

24 Schmelzeisen R, Cienfuegos-Monroy R, Schön R et al. Patient benefit from endoscopically assisted fixation of condylar neck fractures-a randomized controlled trial. J Oral Maxillofac Surg 2009; 67: 147-158

25 Schneider M, Erasmus F, Gerlach KL et al. Open reduction and internal fixation versus closed treatment and mandibulomaxillary fixation of fractures of the mandibular condylar process: a randomized, prospective, multicenter study with special evaluation of fracture level. J Oral Maxillofac Surg 2008; 66: 25372544

26 Schön R, Fakler O, Gellrich NC et al. Five year experience with the transoral endoscopic-assisted treatment of displaced condylar mandible fractures. Plast Reconstr Surg 2005; 116: 44-50

27 Schön R, Gellrich NC, Schmelzeisen R. Minimal invasive open reduction of dislocated pediatric condylar fracture. Brit J Oral Maxillofac Surg 2005; 43: 258-260

${ }^{28}$ Silverman S. A new operation for displaced fractures at the neck of the mandibular condyle. Dental Cosmos 1925; 67: 876-877

${ }^{29}$ Strobl H, Emshoff R, Rothler G. Conservative treatment of unilateral condylar fractures in children: a long-term clinical and radiologic follow-up of 55 patients. Int J Oral Maxillofac Surg 1999; 28: 95-98

30 Thoren H, Hallikainen D, Iizuka T et al. Condylar process fractures in children: a follow-up study of fractures with total dislocation of the condyle from the glenoid fossa. J Oral Maxillofac Surg 2001; 59: 768-774

${ }^{31}$ Thoren H, Iizuka T, Hallikainen D et al. An epidemiological study of patterns of condylar fractures in children. Br J Oral Maxillofac Surg 1997: 35: 306-311

32 Valiati R, Ibrahim D, Abreu ME et al. The treatment of condylar fractures: to open or not to open? A critical review of this controversy. Int J Med Sci 2008; 5: 313-318

33 Veras RB, Kriwalsky MS, Eckert AW et al. Longterm outcomes after treatment of condylar fracture by intraoral access: a functional and radiologic assessment. J Oral Maxillofac Surg 2007; 65: 1470-1476

34 Widmark G, Bagenholm T, Kahnberg KE et al. Open reduction of subcondylar fractures. Int J Oral Maxillofac Surg 1996; 25: 107

\section{Dr. med. Dr. med. dent. Sebastian Schiel}

Klinik für Mund-, Kiefer- und Gesichtschirurgie Klinikum Augsburg Stenglinstraße 2 86165 Augsburg und Praxis für Mund-, Kiefer- und Gesichtschirurgie im Pferseepark Franz-Kobinger-Straße 7 A 86157 Augsburg

Dr. med. dent. Florian Probst

Assistenzarzt

Dr. med. Peter Mayer

Assistenzarzt

Prof. Dr. med. dent.

Gabriele Kaeppler

Leitung Röntgenabteilung und Fachzahnärztin für Oralchirurgie

Prof. Dr. med. Dr. med. dent. Carl Peter Cornelius

Oberarzt

Klinik und Poliklinik für Mund-, Kiefer- und Gesichtschirurgie Klinikum der Universität München Lindwurmstraße 2 a 80337 München

peter.cornelius@med.uni-muenchen. de (E-Mail-Adressat für alle Autoren) 